\title{
The Use of Sensitive Imaging Modalities for Cervical Cancer Staging in Japan
}

\author{
Takahiro Higashi $^{1}$, Tomone Watanabe ${ }^{1}$, Momoko Iwamoto ${ }^{1} \&$ Mikio Mikami $^{2}$ \\ ${ }^{1}$ Division of Health Services Research, Center for Cancer Control and Information Services, National Cancer \\ Center, Tokyo, Japan \\ ${ }^{2}$ Department of Obstetrics and Gynecology, School of Medicine, Tokai University, Kanagawa, Japan \\ Correspondence: Tomone Watanabe, Division of Health Services Research, Center for Cancer Control and \\ Information Services, National Cancer Center, 5-1-1, Tsukiji, Chuo-ku, Tokyo, 104-0045, Japan. Tel: \\ 81-3-3547-5201. E-mail: tomonwat@ncc.go.jp
}

Received: November 29, 2018 Accepted: December 18, 2018 Online Published: February 25, 2019

doi:10.5539/gjhs.v11n3p75 URL: https://doi.org/10.5539/gjhs.v11n3p75

\begin{abstract}
The International Federation of Gynecology and Obstetrics (FIGO) staging system for cervical cancer did not take into account any of the findings determined by imaging modalities as part of the staging work-up. However, in the Japanese clinical settings, computed tomography (CT) and magnetic resonance imaging (MRI) are frequently used In this study, we aimed to describe the pretreatment use of sensitive imaging modalities in Japan in order to assess the future adaptability of the FIGO staging system.Data from September 2012 to December 2014 were collected from the National Database of the Hospital-Based Cancer Registry and health insurance claims data. A total of 280 hospitals participated. From the database, all patients with cervical cancer who received first-line therapy at the participating hospitals were analyzed. The proportions of patients who had CT, MRI, and positron emission tomography-CT (PET-CT) before receiving the first-line therapy were calculated. For comparison, the proportions of patients who had undergone cystoscopy and/or proctoscopy -- examinations that are incorporated into the FIGO system -- were also calculated. A total of 13668 patients were included; $77.3 \%$ of patients had early stage (stage 0 or I) disease. Among all patients, $88.5 \%$ had undergone CT, MRI, or PET-CT before receiving the first-line therapy. Additionally, over $90 \%$ of patients with advanced-stage (stage II-IV) disease had undergone CT. Conversely, only $21.0 \%$ of patients with stage II-IV disease were reported to have undergone cystoscopy and/or proctoscopy. Promoting a resource-stratified approach in the cervical cancer staging is warranted.
\end{abstract}

Keywords: cervical cancer, computed tomography, FIGO staging, magnetic resonance imaging, Positron emission tomography-CT

\section{Introduction}

Cervical cancer is the second most common gynecological malignancy in Japan. In 2010, the age-standardized incidence rate of cervical cancer, including carcinoma in situ, was 52.1 per 100000 , and the mortality rate was 4.1 per 100000 (National Cancer Center [NCC], 2015). Despite the slight increase in the mortality rate in the recent years, it has considerably reduced by 1995 owing to cervical cancer screening and improved treatment strategies (NCC, 2015).

Cancer staging is a vital component of diagnostic evaluation, which enables clinicians to make appropriate treatment choices. In Japan, like most other countries, the International Federation of Gynecology and Obstetrics (FIGO) staging system is in wide use; it was first established in 1958 and most recently updated in 2009 (Pecorelli, Zigliani, \& Odicino, 2009; Pecorelli, 2009). The 2009 FIGO staging system incorporates the information obtained during the primary clinical evaluations, including data on: palpation and inspection of the primary tumor, inguinal and supraclavicular lymph node palpation, colposcopy, endocervical curettage, cervical conization and proctoscopy, or basic imaging tests such as intravenous urography, barium enema, and radiography of the lungs and bones. However, the FIGO staging system of 2009 did not incorporate the findings determined using sensitive imaging modalities such as computed tomography (CT) scans and magnetic resonance imaging (MRI), due to the limited access to such sophisticated imaging equipment in many parts of the world. For this reason, the use of these modalities is encouraged rather as an adjunct examination whenever available. As technology advances, however, greater access to these imaging modalities makes the FIGO staging system appear somewhat obsolete, if not 
completely behind the curve, especially in current clinical practice in resource-abundant countries (Erickson et al., 2017).

The FIGO staging system has long been adopted as the major staging method by the Japan Society of Obstetrics and Gynecology despite the frequent use of imaging modalities in the country. For instance, in 2013, the number of CTs and MRIs performed in Japan were 2.3- and 1.3-times higher respectively than those in the US (OECD, 2014). Patients prognosis requires a realm of clinical information such as nodal status, tumor volume, and depth of invasion, all of which can be examined by sensitive imaging modalities (Bhosale, Peungjesada, Devine, Balachandran, \& Iyer, 2010). Therefore, given the availability of these tools in Japan, it is not difficult to presume that many Japanese clinicians use such tools in determining patients' treatment plans. However, to the best of our knowledge, no report thus far has suggested the visualization of the gap between the status quo and the FIGO classification system. Therefore, this study sought to describe the pretreatment utilization of sensitive imaging modalities in Japan in order to assess the future adaptability of the FIGO staging system in resource-abundant countries.

\section{Materials and Methods}

We used a database created for the quality-of-care assessment project that linked the National Database of the Hospital-Based Cancer Registry (HBCR), and the health insurance claims data from the Diagnosis Procedure Combination/per Diem Payment System (DPC/PDPS) survey submitted from the same hospitals (Higashi et al., 2013; Iwamoto, Nakamura, \& Higashi, 2016). The HBCR is a cancer incidence reporting system for all designated cancer care hospitals (DCCHs) and many non-designated hospitals that play similar roles as DCCHs in their communities. In principle, the HBCR registers all cancer patients visiting the hospitals. The HBCR data contain clinical information including the clinical and pathological stages as well as the tumor-node-metastasis (TNM) classifications, tumor location, and histopathological findings based on the International Classification of Diseases Oncology $3^{\text {rd }}$ edition (ICD-O-3). As part of the quality of care monitoring and improvement at the national level, health insurance claims data from hospitals that adopt HBCRs were collected. The DPC/PDPS was used to determine the per-day reimbursement based on groups defined by diagnoses and procedures performed during hospitalization. Further, data on individual services provided were also collected to monitor the effect of introducing the DPC/PDPS system. Since these data are equivalent to the traditional fee-for-service claims data, for simplicity, we refer to the data as "insurance claims" in this manuscript. These data contain information on all health services provided, including tests, images, procedures, and prescribed drugs, along with the dates and costs of the services from both inpatient and outpatient units for all insurance types. We extracted insurance claims data from September 2012 to December 2014 and linked them to the HBCR data. The details of the data collection processes have been previously described (Iwamoto et al., 2016).

All patients with cervical cancer (ICD-O morphology: C53.0-53.9) were identified from the HBCR. Patients with cervical cancer who had surgery, radiotherapy, chemotherapy, and any combinations of these as the first-line therapy were included in the analysis. Cervical conization was regarded as surgery for it can be the final treatment for patients with stage 0 or IA cervical cancer. The sensitive imaging modalities considered in this study were CT, MRI, and PET-CT. Cystoscopy and proctoscopy -- examinations that are taken into account in the FIGO system -were also included as basic examination procedures for diagnosis and staging. The tumor stage was categorized as 0, I, II, III, IV (IVA/IVB), or unknown according to the FIGO staging system based on the T component of the Union for International Cancer Control (UICC) cancer staging system. Although stage 0 is not included in the FIGO staging system, we included carcinoma in situ (coded as "Tis" in HBCR) in this study due to its high prevalence in Japan. Additionally, according to the HBCR coding rules, cancers that were diagnosed after treatment were coded as having an "unknown" clinical stage. Although HBCR contains data regarding both clinical (pretreatment) and pathological stages, we only considered clinical stages in our analysis.

Stage stratified proportions and their 95\% confidence intervals (CIs) among those who received CT, MRI, and PET-CT scans at the hospital before first-line therapy were calculated. For comparison, we calculated the proportion of patients who received cystoscopy and/or proctoscopy before the first-line therapy. Additionally, we limited the analysis to those who received their first-line treatment at the hospitals where their diagnoses were established. Since patients who were initially diagnosed at a different facility before referral may have undergone diagnostic evaluation at the different facilities, they were excluded from the analysis. All analyses were performed using Stata version 13 (StataCorp LP, College Station, TX, USA).

This study protocol was approved by the institutional review board of the National Cancer Center, Japan. Informed consent was not required for the present study, based on the Ethical Guidelines for Medical Research Involving Human Subjects. 


\section{Results}

A total of 13668 cervical cancer patients from 280 hospitals were included in the study (Table 1).

Table 1. Demographic information of 13668 patients

\begin{tabular}{|c|c|c|c|c|c|c|}
\hline Stage & $\mathbf{0}$ & I & II & III & IV & Unknown \\
\hline & 8314 & 2271 & 1441 & 759 & 280 & 613 \\
\hline \multirow[t]{2}{*}{ Number of Patients, N (\%) } & $(60.8)$ & $(16.6)$ & $(10.5)$ & $(5.5)$ & $(2.0)$ & $(4.5)$ \\
\hline & 40.1 & 49.1 & 58.5 & 63.4 & 65.0 & 43.5 \\
\hline Age, Mean (SD) & $(11.0)$ & $(14.4)$ & $(15.1)$ & $(15.0)$ & $(14.3)$ & $(13.3)$ \\
\hline \multicolumn{7}{|l|}{ First-line treatment, $\mathrm{N}(\%)$} \\
\hline Surgery & $\begin{array}{l}8229 \\
(99.1)\end{array}$ & $\begin{array}{l}1214 \\
(53.5)\end{array}$ & $\begin{array}{l}73 \\
(5.1)\end{array}$ & $\begin{array}{l}2 \\
(0.3)\end{array}$ & $\begin{array}{l}1 \\
(0.4)\end{array}$ & $544(88.7)$ \\
\hline \multirow{3}{*}{ Radiotherapy } & 13 & 158 & 238 & 222 & 90 & 7 \\
\hline & $(0.2)$ & $(7.0)$ & $(16.5)$ & $(29.3)$ & $(32.1)$ & $(1.1)$ \\
\hline & 1 & 16 & 53 & 59 & 29 & 7 \\
\hline \multirow[t]{2}{*}{ Chemotherapy } & $(0.0)$ & $(0.7)$ & $(3.7)$ & $(7.8)$ & $(10.4)$ & $(1.1)$ \\
\hline & 13 & 347 & 275 & 13 & 7 & 16 \\
\hline \multirow[t]{2}{*}{ Surgery and chemotherapy } & $(0.2)$ & $(15.3)$ & $(19.1)$ & $(1.7)$ & $(2.5)$ & $(2.6)$ \\
\hline & 2 & 126 & 558 & 439 & 128 & 7 \\
\hline \multirow[t]{2}{*}{ Radiotherapy and chemotherapy } & $(0.0)$ & $(5.6)$ & $(38.7)$ & (57.8) & $(45.7)$ & $(1.1)$ \\
\hline & 46 & 410 & 244 & 24 & 25 & 32 \\
\hline Other & $(0.6)$ & (18.1) & (16.9) & $(3.2)$ & $(8.9)$ & $(5.2)$ \\
\hline
\end{tabular}

Note. Cervical conization was included in the surgery category as a first-line treatment for stage 0 disease.

Patients with early-stage disease (stage 0 and I) accounted for $77.3 \%$ (10 585 patients) of all. The mean (standard deviation) age was 45.5 (14.8) years, and patients with early-stage disease were younger than those with advanced-stage (stage III and IV) disease. Surgery was the most frequently performed first-line treatment for patients with early-stage disease, and radiation therapy and chemotherapy (including chemo-radiotherapy) were most frequently performed for patients with advanced disease. 

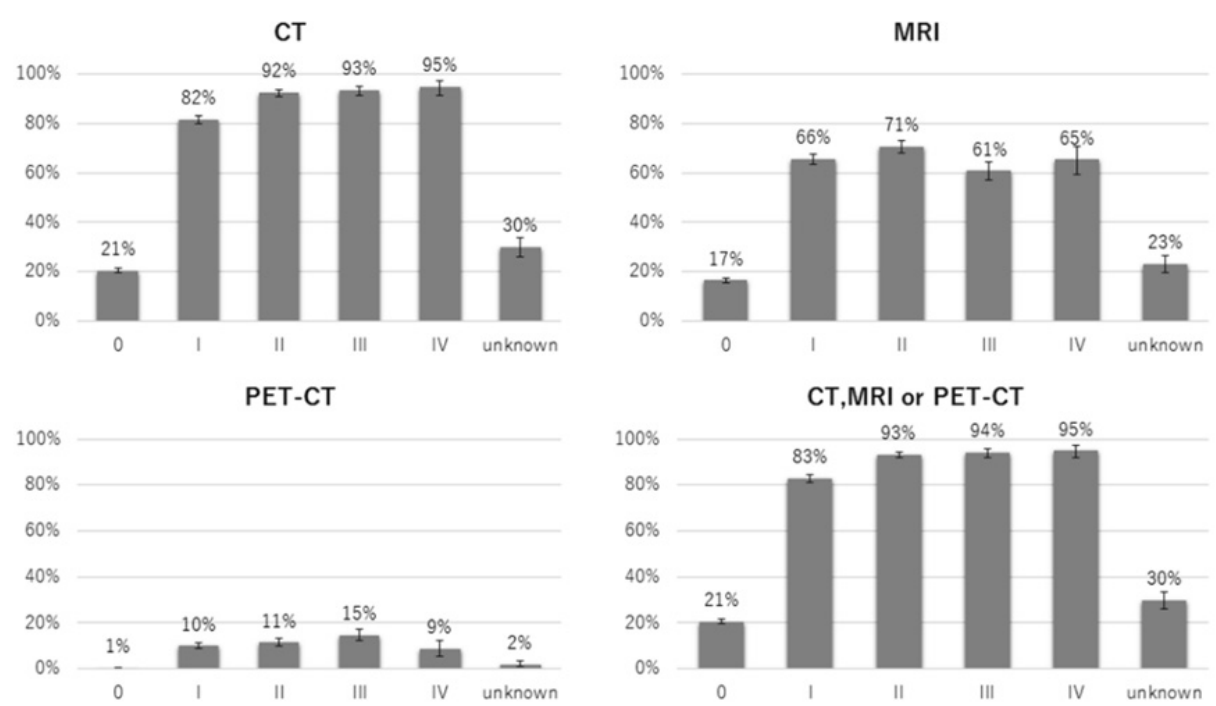

Figure 1. Proportions of cervical cancer patients $(n=13,668)$ assessed using sensitive imaging modalities before first-line treatment, according to the clinical stage

Figure 1 shows the proportions of patients who received imaging evaluations (CT, MRI, and/or PET-CT) before first-line treatments. Overall, $88.5 \%$ (3 800 patients) of all with invasive disease (stage I-IV) had undergone imaging examination, although patients with noninvasive disease (stage 0 ) were examined by these modalities less frequently (20.7\%). CT was the most frequently performed imaging modality before first-line treatment (44.3\%). Among patients with stage II-IV disease, more than $90 \%$ of patients had undergone CT. PET-CT was less frequently used; only $12.1 \%$ of patients with stage II-IV disease had undergone PET-CT.

Of all subjects, 9378 (68.6\%) patients were diagnosed and treated at the same hospital (Table 2).

Table 2. Demographic information of 9388 patients

\begin{tabular}{|c|c|c|c|c|c|c|}
\hline Stage & $\mathbf{0}$ & I & II & III & IV & Unknown \\
\hline & 5945 & 1292 & 903 & 463 & 208 & 577 \\
\hline \multirow[t]{2}{*}{ Number of Patients, N (\%) } & $(63.3)$ & $(13.8)$ & $(9.6)$ & (4.9) & $(2.2)$ & $(6.1)$ \\
\hline & 40.5 & 50.4 & 58.6 & 63.4 & 65.5 & 42.8 \\
\hline Age, Mean (SD) & $(11.1)$ & $(14.4)$ & $(15.3)$ & $(14.8)$ & $(14.7)$ & $(12.6)$ \\
\hline \multicolumn{7}{|l|}{ First-line treatment, N(\%) } \\
\hline & 5883 & 659 & 45 & 1 & 1 & 520 \\
\hline \multirow[t]{2}{*}{ Surgery } & $(99.1)$ & $(51.0)$ & $(5.0)$ & $(0.2)$ & $(0.5)$ & $(90.1)$ \\
\hline & 6 & 93 & 133 & 125 & 69 & 4 \\
\hline \multirow[t]{2}{*}{ Radiotherapy } & $(0.1)$ & $(7.2)$ & $(14.7)$ & $(27.0)$ & $(33.2)$ & $(0.7)$ \\
\hline & 1 & 12 & 31 & 40 & 19 & 5 \\
\hline \multirow[t]{2}{*}{ Chemotherapy } & $(0.0)$ & $(0.9)$ & (3.4) & $(8.6)$ & $(9.1)$ & $(0.9)$ \\
\hline & 7 & 209 & 164 & 9 & 6 & 13 \\
\hline \multirow[t]{2}{*}{ Surgery and chemotherapy } & $(0.1)$ & $(16.2)$ & $(18.2)$ & $(1.9)$ & $(2.9)$ & $(2.3)$ \\
\hline & 2 & 67 & 367 & 273 & 93 & 5 \\
\hline \multirow[t]{2}{*}{ Radiotherapy and chemotherapy } & $(0.0)$ & (5.2) & $(40.6)$ & $(59.0)$ & $(44.7)$ & (0.9) \\
\hline & 36 & 252 & 163 & 15 & 20 & 30 \\
\hline Other & (0.6) & (19.5) & $(18.1)$ & (3.2) & (9.6) & (5.2) \\
\hline
\end{tabular}

Note. Cervical conization was included in surgery as the first-line treatment for Stage 0 disease. 
The characteristics of these patients were similar to those from the overall cohort. Figure 2 shows the proportions of patients who received imaging evaluations (CT, MRI, and/or PET-CT) before first-line treatments among this cohort. Among this limited group of patients, $90.7 \%$ (2 599 patients) with stage I-IV disease had imaging examinations performed. The proportions of patients who received CT and MRI before first-line treatment were slightly higher than those among the overall cohort.
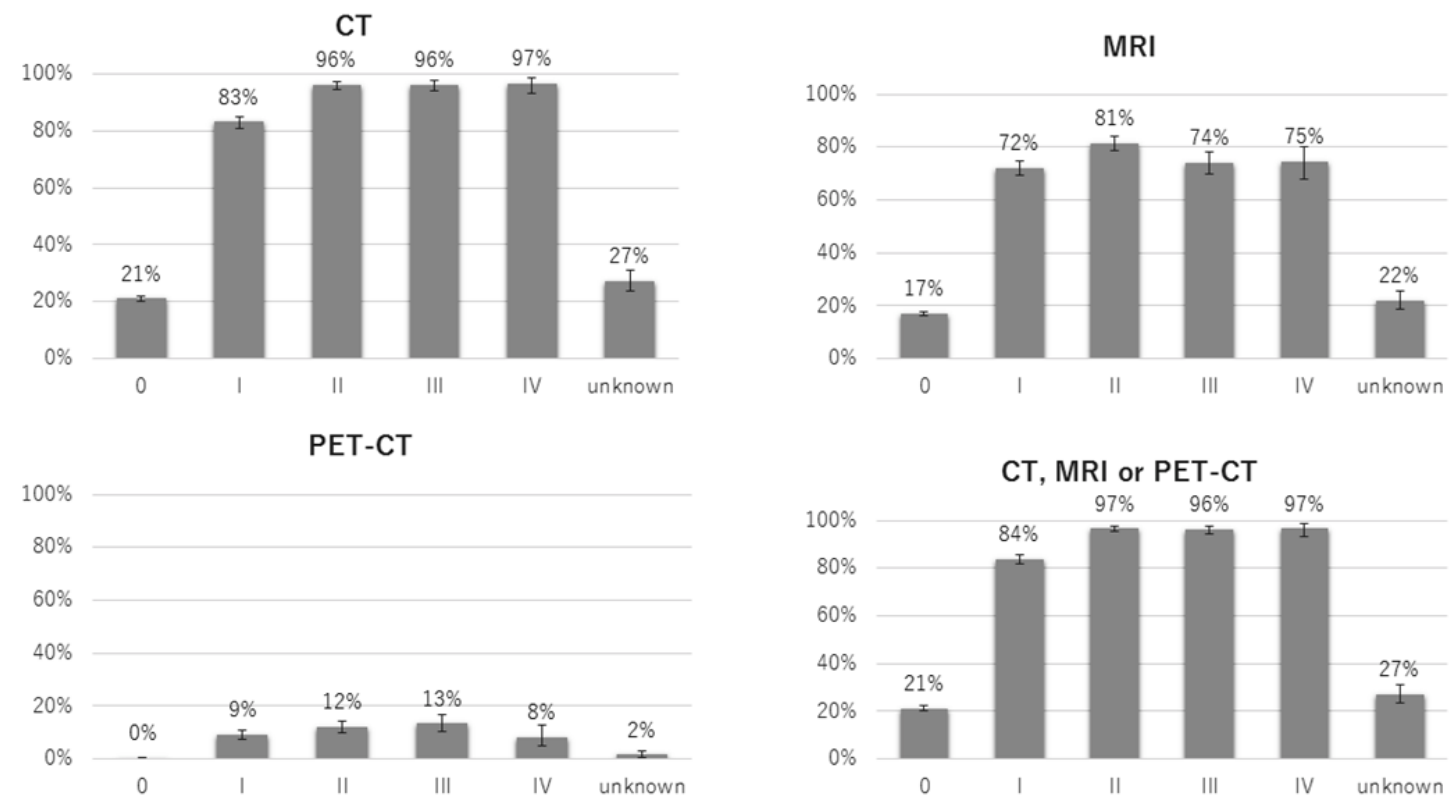

Figure 2. Proportions of cervical cancer patients $(n=9,388)$, diagnosed and treated at the same hospital, assessed using sensitive imaging modalities before first-line treatment, according to the clinical stage

Table 3 shows the proportions of patients who received cystoscopy and/or proctoscopy before first-line treatment. The use of cystoscopy and/or proctoscopy was less frequent than CT/MRI -- only $21.0 \%$ of patients with invasive cancer underwent cystoscopy and/or proctoscopy as part of their work-up. Further, only $33.3 \%$ of patients with stage IVA disease (invasion of the bladder or rectum mucosa as a staging criterion) had undergone cystoscopy and/or proctoscopy, while $96.8 \%$ of these patients received CT and/or MRI.

Table 3. Proportions of patients who underwent cystoscopy and/or proctoscopy before initiating first-line treatment

\begin{tabular}{lcccccc}
\hline Stage & $\mathbf{0}$ & I & II & III & IV & Unknown \\
\hline \multirow{2}{*}{ Underwent/overall cohort, ${ }^{a} \mathrm{n}(\%)$} & $21 / 8304$ & $203 / 2271$ & $231 / 1441$ & $189 / 759$ & $101 / 280$ & $12 / 613$ \\
& $(0.3)$ & $(8.9)$ & $(16.0)$ & $(24.9)$ & $(36.1)$ & $(2.0)$ \\
\hline \multirow{2}{*}{ Underwent/limited cohort, ${ }^{\mathrm{b}} \mathrm{n}(\%)$} & $17 / 5935$ & $128 / 1292$ & $157 / 903$ & $126 / 463$ & $77 / 208$ & $6 / 577$ \\
& $(0.3)$ & $(9.9)$ & $(17.4)$ & $(27.2)$ & $(37.0)$ & $(1.0)$ \\
\hline
\end{tabular}

${ }^{\mathrm{a}}$ All patients included in this study $(\mathrm{n}=13,668) ;{ }^{\mathrm{b}}$ Only patients who were diagnosed and treated at the same hospital $(\mathrm{n}=9,378)$.

\section{Discussion}

In this study, we found that majority of Japanese patients with stage I-IV cervical cancer received imaging evaluations before their first-line treatments. CT was among the most frequently used imaging modalities (88\%), and more than $75 \%$ of the patients had undergone MRI. The frequent use of sensitive radiological imaging modalities supports the idea that these modalities are well-established methods for initial evaluation of cervical cancer patients in Japan. In contrast, non-radiological examinations such as cystoscopy and proctoscopy were less frequently used. This fact may imply that Japanese gynecologists value findings determined using sensitive 
radiological imaging during the pre-treatment work-up although the findings are not incorporated in the FIGO staging system.

Theoretically, a well-established cancer staging system enables an appropriate stratification of treatment planning based on an accurate prediction of prognosis. However, as mentioned above, the FIGO staging system of 2009 did not incorporate lymph node status and parametrial involvement, which might have influenced the survival and prognosis, despite the fact that these factors can be well-visualized using sensitive imaging modalities. In reality, however, sensitive imaging modalities are in wide use, influencing the detection of the stages. For example, the gynecologic cancer registry operated by the Japan Society of Obstetrics and Gynecology once reported that $25 \%$ of stage IVB cervical cancer patients were classified into this stage based only on the findings of paraaortic lymph node involvement (Japan Society of Obstetrics and Gynecology, 2017). Incoherent use of imaging modalities in the staging practice could lead to discrepancies in staging and the choice of treatments.

The cancer staging naturally reflects the availability of sensitive imaging techniques. In the United States, the American Society of Clinical Oncology recently proposed resource-stratified guidelines for cervical cancer in order to address the disparities in country-specific resources (Chuang et al., 2016). In the same manner, the FIGO staging system, which works vis-à-vis existing treatment guidelines, could also be revised in order to incorporate the findings of imaging modalities whenever available.

Previous studies have shown that MRI is a more suitable a method than CT in detecting the primary tumor and parametrial invasion (Bipat et al., 2003; Koyama, Tamai, \& Togashi, 2007; Ozsarlak et al., 2003). Our study showed that while CT is more frequently used, the majority of patients were assessed using both CT and MRI. Although disregarded by the 2009FIGO staging system, MRI and CT have compelling accuracies in detecting nodal metastasis, which greatly affects ones' treatment plan. Conversely, CT and MRIare not as suitable in detecting micrometastases in normal-sized nodes (Yang, Lam, Yu, Cheung, \& Metreweli, 2000). PET-CT has also been reported to be the best modality to detect nodal metastasis in patients with cervical cancer (Dalla Palma et al., 2012; Iyer \& Devine, 2007; Liyanage, Roberts, \& Rockall, 2010). The National Comprehensive Cancer Network suggests using PET-CT for pretreatment evaluations of patients with stage IB-II or higher cervical cancer (National Comprehensive Cancer Network [NCCN], 2016). Since PET-CT is less readily available than CT and MRI in Japan, some patients are referred to other hospitals for PET-CT examination. Our data do not capture these services provided by facilities other than those that provided the main treatment. Thus, our results likely underestimate the actual proportion of patients undergoing PET-CT for treatment planning. Future studies examining the utility of PET-CT and investigating services performed in referral facilities are warranted.

Our study also found that approximately $20 \%$ of patients with stage 0 disease received CT before first-line treatment despite the low risks of parametrial invasion and metastases. While using the sensitive imaging modalities is ideal for staging and treatment selection, excessive use is not desirable as it can cause unnecessary risk of radiation exposure as well as higher costs. Such excessive use may be attributed to an obsession to confirm the absence of metastasis, fear of litigation, mindless repetition of the established routine, and patients' expectations (Mendelson, 2010).

This study was subject to several limitations. First, data were collected predominantly from DCCHs (93.9\%). Patients treated in small community hospitals may be less likely to receive imaging modalities as part of their pretreatment work-ups. However, DCCHs are estimated to cover up to $68.1 \%$ of all cancer patients in Japan, and a similar trend may be predicted for non-DCCHs (NCC, 2015). Second, insurance claims data were obtained only from the participating hospitals. Thus, our data do not capture health services provided at other hospitals even though they could have gone through a series of treatment at different hospitals. For instance, since PET-CT is less readily available than CT and MRI in Japan, some patients are referred to other hospitals for PET-CT examination. Our results therefore might underestimate the frequency of use of these imaging modalities. Third, we could not distinguish cervical conization as a component of the work-up from the treatment - we regarded all conization as treatment in this study. This should be considered when interpreting the treatment distribution.

\section{Conclusion}

Despite these limitations, our study revealed that most patients with invasive cervical cancer received sensitive imaging before starting first-line treatment in Japan. This clinical practice visualizes the difference between the status quo and what was recommended in the FIGO staging system -- which considered these imaging modalities to be options rather than requirements. Thus, promoting resource-stratified management in the cervical cancer staging system, which incorporates the sensitive imaging modalities in the FIGO staging system, is essential. Whenever developing international standards of practice, studies aimed at designing and introducing a resource-stratified staging system are warranted. 


\section{Acknowledgements}

We thank Kaoru Konno for her technical assistance in conducting the research. This study was supported by National Cancer Center, Japan.

\section{Competing Interests Statement}

The authors declare that there are no competing or potential conflicts of interest.

\section{References}

Bhosale, P., Peungjesada, S., Devine, C., Balachandran, A., \& Iyer, R. (2010). Role of Magnetic Resonance Imaging as an Adjunct to Clinical Staging in Cervical Carcinoma. J Comput Assist Tomogr, 34(6), 855-864. https://doi.org/10.1097/RCT.0b013e3181ed3090

Bipat, S., Glas, A. S., van der Velden, J., Zwinderman, A. H., Bossuyt, P. M., \& Stoker, J. (2003). Computed Tomography and Magnetic Resonance Imaging in Staging of Uterine Cervical Carcinoma: A systematic review. Gynecol Oncol, 91(1), 59-66.

Center for Cancer Control and Information Services, N. C. C. (2015). Cancer statistics in Japan. Retrieved from http://ganjoho.jp/en/professional/statistics/table_download.html

Center for Cancer Control and Information Services, N. C. C. (2015). Aggregate data report of the national database of the hospital-based cancer registry for designated cancer care hospitals for 2013. Retrieved from https://ganjoho.jp/data/reg_stat/statistics/brochure/2013_report.pdf

Chuang, L. T., Temin, S., Camacho, R., Duenas-Gonzalez, A., Feldman, S., Gultekin, M., . . Berek, J. S. (2016). Management and Care of Women With Invasive Cervical Cancer: American Society of Clinical Oncology Resource-Stratified Clinical Practice Guideline. $J$ Glob Oncol, 2(5), 311-340. https://doi.org/10.1200/jgo.2016.003954

Dalla Palma, M., Gregianin, M., Fiduccia, P., Evangelista, L., Cervino, A. R., Saladini, G., . . Zagonel, V. (2012). PET/CT Imaging in Gynecologic Malignancies: A critical overview of its clinical impact and our retrospective single center analysis. Crit Rev Oncol Hematol, 83(1), 84-98. https://doi.org/10.1016/j.critrevonc.2011.10.002

Development, O. f. E. C.-o. a. (2014). OECD Health Statistics 2014. Retrieved from http://www.oecd.org/els/health-systems/oecd-health-statistics-2014-frequently-requested-data.htm

Erickson, A. B., Olawaiye, A. B., Bermudez, A., Bhosale, P. R., Grendys, E. C., Grigsby, P. W., . . Mutch, D. G. (2017). Cervix Uteri AJCC Cancer Staging Manual, Eighth edn. (pp.649-659). Chicago: Springer.

Higashi, T., Nakamura, F., Shimada, Y., Shinkai, T., Muranaka, T., Kamiike, W., . . Okamura, T. (2013). Quality of Gastric Cancer Care in Designated Cancer Care Hospitals in Japan. Int J Qual Health Care, 25(4), 418-428. https://doi.org/10.1093/intqhc/mzt041

Iwamoto, M., Nakamura, F., \& Higashi, T. (2016). Monitoring and Evaluating the Quality of Cancer Care in Japan Using Administrative Claims Data. Cancer Sci, 107(1), 68-75. https://doi.org/10.1111/cas.12837

Iyer RB, B. A., \& Devine, C. E. (2007). PET/CT and Cross Sectional Imaging of Gynecologic Malignancy. Cancer Imaging, (7), S130-138. https://doi.org/10.1102/1470-7330.2007.9015

Japan Society of Gynecologic Oncology. (2017). Instruction for the clinical stage classification and cancer registry for cervical cancer. Retrieved from http://fa.kyorin.co.jp/jsog/readPDF.php?file=69/5/069051361.pdf

Koyama, T., Tamai, K., \& Togashi, K. (2007). Staging of Carcinoma of the Uterine Cervix and Endometrium. Eur Radiol, 17(8), 2009-2019. https://doi.org/ MRI and PET scans for primary staging and detection of cervical cancer recurrence. https://doi.org/10.1007/s00330-006-0555-0

Liyanage, S. H., Roberts, C. A., \& Rockall, A. G. (2010). MRI and PET Scans for Primary Staging and Detection of Cervical Cancer Recurrence. Womens Health (Lond), 6(2), 251-267; quiz 268-259. https://doi.org/10.2217/whe.10.7

Mendelson RM, B. P. (2010). Inappropriate Imaging: why it matters, why it happens, what can be done. $J$ Med Imaging Radiat Oncol, 54(3), 173-177. https://doi.org/10.1111/j.1754-9485.2010.02173.x

Network, N. C. C. (2015). NCCN practice guidelines in oncology (NCCN Guideline) cervical cancer version I. 2016. Retrieved from https://www.nccn.org/professionals/physician_gls/PDF/cervical.pdf 
Ozsarlak, O., Tjalma, W., Schepens, E., Corthouts, B., Op de Beeck, B., Van Marck, E., .. . De Schepper, A. M. (2003). The Correlation of Preoperative CT, MR imaging, and Clinical Staging (FIGO) with Histopathology Findings in Primary Cervical Carcinoma. Eur Radiol, 13(10), 2338-2345. https://doi.org/10.1007/s00330-003-1928-2

Pecorelli, S. (2009). Revised FIGO Staging for Carcinoma of the Vulva, Cervix, and Endometrium. Int J Gynaecol Obstet, 105(2), 103-104.

Pecorelli, S., Zigliani, L., \& Odicino, F. (2009). Revised FIGO Staging for Carcinoma of the Cervix. Int $J$ Gynaecol Obstet, 105(2), 107-108. https://doi.org/10.1016/j.ijgo.2009.02.009

Yang, W. T., Lam, W. W., Yu, M. Y., Cheung, T. H., \& Metreweli, C. (2000). Comparison of Dynamic Helical CT and Dynamic MR Imaging in the Evaluation of Pelvic Lymph Nodes in Cervical Carcinoma. AJR Am J Roentgenol, 175(3), 759-766. https://doi.org/10.2214/ajr.175.3.1750759

\section{Copyrights}

Copyright for this article is retained by the author(s), with first publication rights granted to the journal.

This is an open-access article distributed under the terms and conditions of the Creative Commons Attribution license (http://creativecommons.org/licenses/by/4.0/). 